\title{
Nuevo método de evaluación de contenidos prácticos en la docencia universitaria
}

Eva Miedes ${ }^{a}$ y Lucía Jordáb

a,b Universidad Politécnica de Madrid. E.T.S.I. Agronómica, Alimentaria y de Biosistemas. Dpto. Biotecnología-Biología Vegetal. Avenida Complutense, 3, 28040. Madrid. SPAIN. eva.miedes@upm.es, lucia.jorda@upm.es

\begin{abstract}
The practical contents in the University subjects are essential for an effective learning of taught theoretical concepts. The evaluation of the learning results of these practices involves, in some subjects, difficulties such as plagiarism and undue dedication of the teacher. This work presents a teaching innovation experience that automates evaluation, avoids plagiarism, promotes continuous practical learning, improves the students' scores and their assessment of the subject.
\end{abstract}

Keywords: automation, schematization, self-evaluation, plagiarism and teaching efficiency

\footnotetext{
Resumen

Los contenidos prácticos en las asignaturas universitarias son fundamentales para un aprendizaje efectivo de los conceptos teóricos impartidos. La evaluación de los resultados de aprendizaje de estas prácticas entraña, en algunas asignaturas, dificultades como plagios y una indebida dedicación por parte del profesor. En este trabajo se presenta una experiencia de innovación educativa que automatiza la evaluación, evita el plagio, promueve el aprendizaje práctico continuo, mejora las calificaciones de los estudiantes y su valoración de la asignatura.
}

Palabras clave: automatización, esquematización, autoevaluación, plagios y eficiencia docente. 


\section{Introducción}

Contexto de aplicación del nuevo método de evaluación de contenidos prácticos en la docencia universitaria.

La implementación del nuevo método de evaluación de los contenidos prácticos se llevó acabo en la Universidad Politécnica de Madrid, entre la Escuela Técnica Superior de Ingeniería de Telecomunicación (http://www.etsit.upm.es/) (teoría) y la Escuela Técnica Superior de Ingeniería Agronómica, Alimentaria y de Biosistemas (http://www.etsiaab.upm.es/) (prácticas).

El proyecto de innovación docente se desarrolló específicamente en la parte práctica de la asignatura de Bioquímica Estructural, que se imparte en el segundo semestre del primer curso del Grado de Ingeniería Biomédica (http://www.etsit.upm.es/estudios/grado-en-ingenieriabiomedica.html), durante los cursos 2014-2015 y 2015-2016. Los alumnos que cursan este Grado cuentan con unas de las notas de acceso más altas de la Universidad Politécnica de Madrid. Se trata de una asignatura obligatoria de 6 ECTS, de los que 1 ECTS pertenecen a la parte práctica. Esta parte consta de dos sesiones teóricas de técnicas instrumentales de bioquímica (4h), dos sesiones teóricas y de problemas de explicación de los fundamentos de las prácticas (3h) y cinco prácticas desarrolladas en tres sesiones en el laboratorio, de cuatro horas cada una (12h). En el curso 2014-2015 y anteriores, la evaluación de esta parte práctica se realizó mediante la media de dos actividades evaluables. La primera consistió en la entrega de un cuaderno de resultados individual por cada una de las cinco prácticas (71 alumnos x 5 prácticas $=355$ cuadernos de prácticas), que se entregaba en papel y en el departamento una semana después de realizar cada práctica. Y la segunda, fue un examen tipo test verdadero/falso (V/F) de 40 preguntas que se realizaba junto con el examen teórico final de la asignatura.

\section{Identificación de puntos de mejora}

Al finalizar el curso 2014-2015, se identificaron, tras la auto-evaluación de la parte práctica de la asignatura, varios aspectos mejorables de la misma. El primero fue la necesidad de reducir el excesivo tiempo de corrección de los cuadernos de resultados. Eran unos 500 cuadernos y el tiempo disponible para la corrección era reducido, ya que los cuadernos eran devueltos a los alumnos con las correcciones oportunas antes del examen final. Esto reducía la posibilidad de implementar nuevas metodologías y mejorar la docencia ya que el tiempo estaba comprometido por las correcciones (Sabagh y Saroyan, 2014). El segundo punto de mejora que se identificó fue el desaprovechamiento de los alumnos de las 12 horas presenciales de prácticas en el laboratorio. Se observó que, aunque el estudiante había recibido una clase de explicación de fundamentos y protocolos con anterioridad, no tenían preparados los contenidos de las mismas y perdían el tiempo intentando comprenderlos en el laboratorio. El tercer punto de mejora se centró en reducir el elevado número de plagios y copias parciales de los cuadernos de resultados (Cronan et al., 2018). Los plagios se identificaron por repetición de errores poco habituales y peculiares que demostraban la copia. En este punto no se usó ninguna de las herramientas de detección de plagios que ofrece la

(cc) EY-NC-ND 2018, Universitat Politècnica de València

Congreso IN-RED (2018) 
UPM como Turnitin (http://turnitin.com/es/) al tratarse de 355 documentos manuscritos. El cuarto aspecto de mejora se centró en aumentar las calificaciones respecto al curso anterior. Los alumnos habían reducido sus notas respecto al curso 2013-2014 debido a la reducción de las notas correspondientes a las prácticas. Y el último aspecto a mejorar, fue la valoración de la asignatura. En la encuesta final de la asignatura, los estudiantes habían evaluado peor que al año anterior la asignatura debido a que consideraban elevada la dedicación a la parte práctica de la misma. Si bien, al inicio de curso, se les había explicado la justa dedicación que debían hacer a la parte práctica (horas) siguiendo los criterios que indica el EEES (www.eees.es).

\section{Objetivos}

Los objetivos del nuevo método de evaluación de contenidos prácticos en la docencia universitaria presentado aquí son: 1) Reducir el tiempo de corrección de los cuadernos de resultados y exámenes; 2) aumentar la preparación del estudiante previa a las sesiones presenciales de prácticas; 3 ) evitar copias en una de las pruebas de evaluación (cuadernos de resultados); 4) mejorar el aprendizaje práctico continuo y las calificaciones y 5) mejorar la valoración de la asignatura por parte de los alumnos.

\section{Desarrollo de la innovación}

Al terminar el curso 2014-2015 se realizó la autoevaluación de la asignatura y se identificaron varios puntos de mejora. Para solucionar estos aspectos mejorables descritos anteriormente, se diseñaron una serie de actuaciones. En la elección y diseño de las actuaciones se priorizó el aprendizaje práctico continuo y la evaluación continua del estudiante, debido a la demostrada eficiencia de los mismos (Seitz, 2018).

Las distintas actuaciones que se detallan a continuación conforman un proyecto de innovación educativa donde se presenta una nueva metodología de evaluación de los contenidos prácticos en la docencia universitaria. La primera de las actuaciones se centró en mejorar la preparación previa (contenidos y fundamentos) del estudiante a las sesiones prácticas. Para ello, los alumnos debían entregar, al comenzar cada sesión práctica, un esquema del protocolo/guion de la(s) práctica(s) a realizar ese día y completar un test de 5 preguntas relativo a la sesión. Dicho test se corregía durante la sesión y se devolvía al estudiante antes de finalizar la sesión para que fuera corregido entre todos. Además, los estudiantes debían completar el cuaderno de resultados en la misma sesión práctica pero no lo entregaban, algo que antes hacían una semana después. Los resultados recogidos en este cuaderno eran importantes ya que posteriormente constituían parte de la materia de la que eran evaluados. La segunda de las actuaciones se centró en reducir el tiempo de corrección por parte de los profesores de los aproximadamente 355 cuadernos de prácticas. Para ello, se optó por una evaluación continua on-line, más automatizada y completa empleando la plataforma de teleenseñanza Moodle-UPM de la asignatura (https://moodle.upm.es/titulaciones/oficiales/). Se diseñó un cuestionario de evaluación por 
cada una de las tres sesiones prácticas que los alumnos completaban tres días después de realizar la sesión práctica. Cada uno de los cuestionarios contaba con 10 preguntas de V/F, 5 preguntas de respuesta múltiple sobre errores prácticos, 2 preguntas tipo gráfica/figura/tabla sobre los resultados obtenidos y recogidos en el cuaderno de prácticas y 3 preguntas sobre datos numéricos procesados (con unidades) obtenidos por cada grupo en las prácticas. Para incluir esos valores numéricos, los estudiantes habían tenido que completar correctamente el cuaderno de resultados en las prácticas o trabajarlo en casa. El cuestionario tenía una duración determinada de entre 8-12 minutos y estaba abierto en una franja horaria acordada previamente con la clase. Al terminar el cuestionario, el estudiante obtenía una evaluación automática de las preguntas contestadas correcta e incorrectamente, así como una retroalimentación explicativa de todas las preguntas. Los resultados numéricos de cada uno de los cuestionarios se obtenían directamente en un archivo de datos y además se añadían a las calificaciones individuales en la plataforma que aloja los espacios virtuales de las asignaturas (Moodle UPM). En resumen, se sustituyó la entrega del cuaderno de resultado de las prácticas por una evaluación continua automatizada on-line del mismo y se eliminó el examen final de prácticas.

Una vez descritas las actuaciones, se diseñaron e implementaron en la plataforma educativa. Seguidamente, se incorporaron como nueva metodología de evaluación en la guía docente del curso siguiente. En la primera clase de presentación de la asignatura, se les explicó a los alumnos el nuevo método de evaluación. Por último, tras aplicar los cambios durante el semestre, se evaluaron los resultados del método de evaluación aplicado en ambos cursos. Para ello se usaron las calificaciones de la parte práctica de la asignatura y varias cuestiones de las encuestas anónimas que los alumnos completan sobre la asignatura y el profesorado.

\section{Resultados}

Los resultados de la aplicación del nuevo método de evaluación de contenidos prácticos en la docencia universitaria se obtuvieron mediante dos indicadores objetivos como son: 1) el análisis comparativo de las calificaciones de los estudiantes de los cursos 2014-2015 (en adelante 2015) y 2015-2016 (en adelante 2016) y 2) el análisis comparativo de los resultados de la encuesta de la asignatura por parte de los alumnos de ambos años.

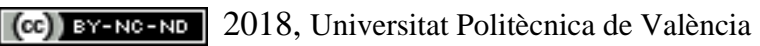

Congreso IN-RED (2018) 


\section{Calificaciones de los estudiantes}

A continuación, se presenta el análisis comparativo de los resultados (calificaciones) obtenidos por los alumnos en la parte práctica de la asignatura (Figura 1).

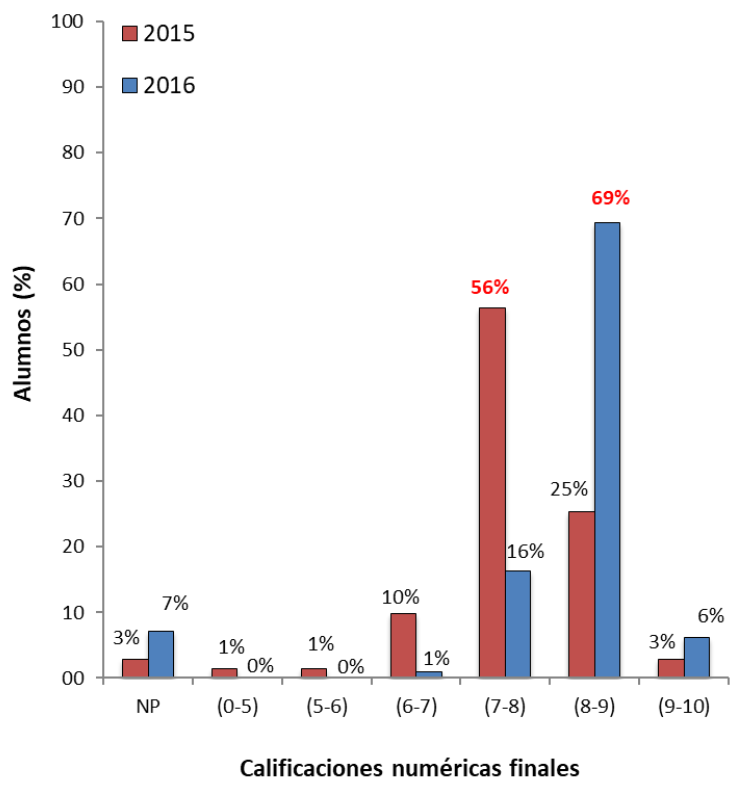

Fig. 1 Comparación de las calificaciones obtenidas por los alumnos de la parte práctica de la asignatura Bioquímica Estructural en los años $2015(n=71)$ y 2016 (n=98). NP: No Presentado.

Como muestra la Figura 1, las calificaciones de los alumnos han mejorado significativamente en el curso 2016 respecto al curso 2015. Los alumnos con notas entre 5 y 8 se han reducido en el curso 2016, mientras que se han incrementado los que alcanzaron una nota entre 8 y 9 . Por lo tanto, la media se ha incrementado un punto y el porcentaje de alumnos con dicha nota ha crecido un 13\%. Los alumnos con calificaciones excelentes (9 y 10) aumentaron un $3 \%$. Los estudiantes no presentados se incrementaron un $4 \%$ en el curso 2016. Esto fue debido al aumento de alumnos Erasmus o bien a otros que pidieron el traslado de Grado. Cabe destacar que las calificaciones no mostraron diferencias significativas entre los cuatro grupos de prácticas (Figura 2). 

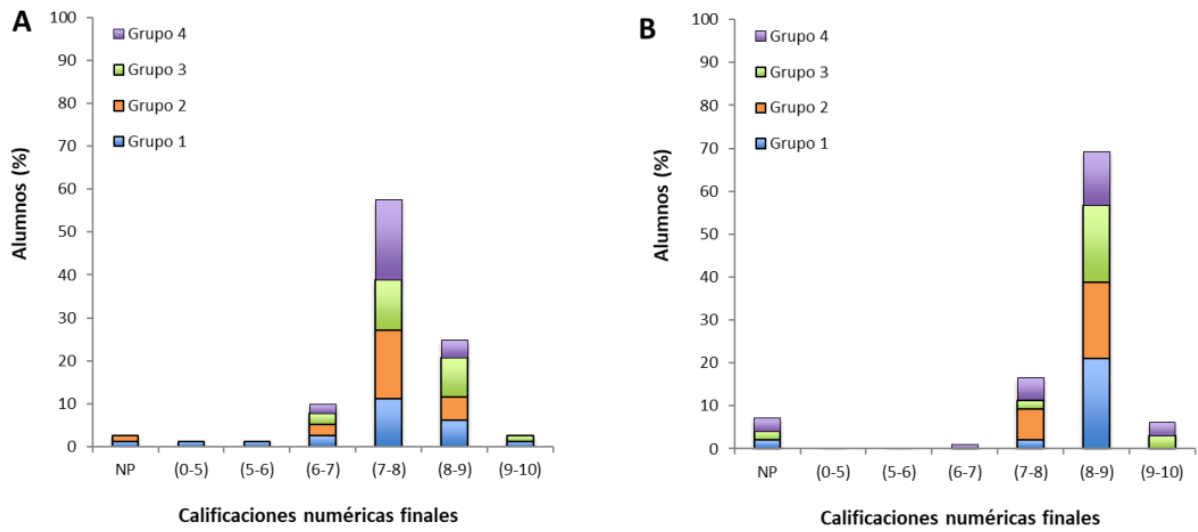

Fig. 2 Comparación de las calificaciones obtenidas por los alumnos de la parte práctica de la asignatura Bioquímica Estructural en los años (A) 2015 (n= 71) y (B) 2016 (n=98) distribuida por grupos.

\section{Evaluación de la parte práctica de la asignatura por parte de los estudiantes}

El análisis comparativo de los resultados de la encuesta de los alumnos de la parte práctica de la asignatura Bioquímica Estructural y de la profesora de prácticas (que fue la misma ambos cursos evaluados), se muestran en la Figura 3. 


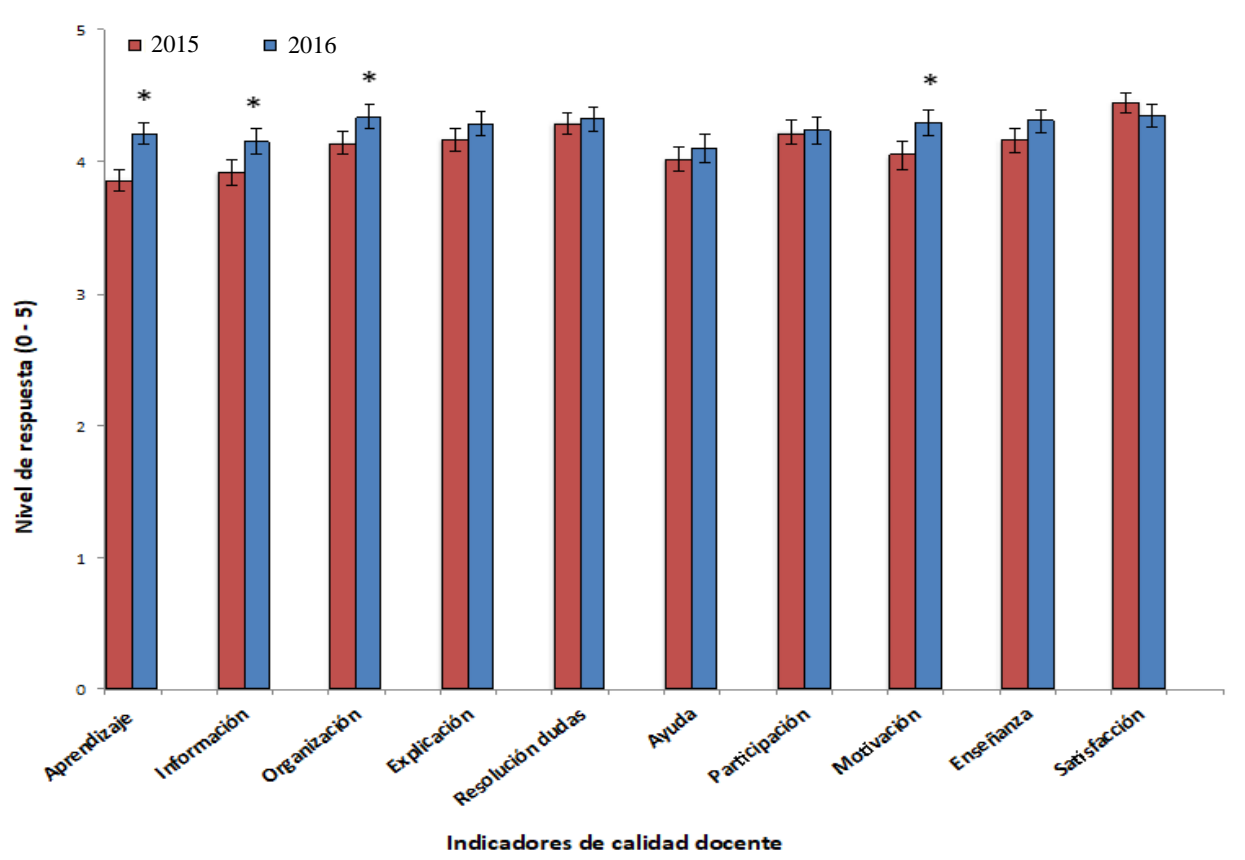

Fig. 3 Comparación de los resultados de la encuesta de los alumnos de la parte práctica de la asignatura Bioquímica Estructural en los años $2015(n=63)$ y $2016(n=74)$. El asterisco (*) muestra diferencias significativas ( $t$-test, $p$-valor $\leq 0.05$ ) entre ambos cursos.

Los resultados de la encuesta de valoración de la asignatura y la profesora han mejorado en el curso 2016 en 9 de los 10 parámetros de calidad que se representan (Figura 3). Se registraron valores significativamente mayores en el año 2016 respecto al 2015 en relación al aprendizaje de los alumnos, información de la asignatura, organización de la asignatura y motivación de los alumnos. Además, la asignatura y la profesora de prácticas mostró unas calificaciones por encima de la media registrada del resto de profesores del Grado de Ingeniería Biomédica (datos no mostrados).

\section{Conclusiones}

Se ha diseñado, aplicado y analizado un nuevo método de evaluación de los contenidos prácticos en la docencia universitaria. Con la puesta en marcha de este proyecto de innovación educativa se han mejorado los puntos críticos identificados. De este modo, se redujo el tiempo de corrección de las pruebas de evaluación y se evitaron los plagios/copias en las mismas. Se aumentó la preparación previa de los estudiantes en contenidos/fundamentos prácticos, lo que mejoró el aprendizaje práctico continuo y elevó las calificaciones. Por último, los estudiantes valoraron y apreciaron muy positivamente el nuevo método de evaluación y, en consecuencia, la valoración general de la asignatura. Con todo ello, las profesoras de la asignatura concluyen que los cambios introducidos mejoran y corrigen significativamente los aspectos problemáticos identificados previamente. 


\section{Referencias}

CRONAN, TP; MULLINS, JK y DOUGLAS, DE. (2018). "Further Understanding Factors that Explain Freshman Business Students' Academic Integrity Intention and Behavior: Plagiarism and Sharing Homework" en Journal of Business Ethics, vol. 147, issue 1, pp. 197-220.

ESCUELA TECNICA SUPERIOR DE INGENIERIA AGRONOMICA, ALIMENTARIA Y DE BIOSISTEMAS. Página general. <http://www.etsiaab.upm.es/> [Consulta: 3 de mayo de 2018] [institucional]

ESCUELA TÉCNICA SUPERIOR DE INGENIEROS DE TELECOMUNICACIONES. Grado en Ingeniería biomédica. <http://www.etsit.upm.es/estudios/grado-en-ingenieria-biomedica.html> [Consulta: 3 de mayo de 2018] [institucional]

ESCUELA TÉCNICA SUPERIOR DE INGENIEROS DE TELECOMUNICACIONES. Página general. <http://www.etsit.upm.es/> [Consulta: 3 de mayo de 2018] [institucional]

ESPACIO EUROPEO DE EDUCACION SUPERIOR. Página general. <http://www.eees.es/> [Consulta: 3 de mayo de 2018] [institucional]

SABAGH, Z y SAROYAN, A. (2014). "Professors' perceived barriers and incentives for teaching improvement" en International Education Research, vol. 2, issue 3, p. 18-30.

SEITZ, AR. (2018). "A New Framework of Design and Continuous Evaluation to Improve Brain Training" en Journal of Cognitive Enhancement, vol. 2, issue 1, pp. 78-87.

TURNITIN. Servicios para la mitigación de riesgo del plagio académico y profesional. <http://turnitin.com/es/> [Consulta: 3 de mayo de 2018]

UNIVERSIDAD POLITÉCNICA DE MADRID. Moodle, herramienta de gestión del aprendizaje para estudios oficiales. <https://moodle.upm.es/titulaciones/oficiales/> [Consulta: 3 de mayo de 2018] [institucional]

(cc) EY-NC-ND 2018, Universitat Politècnica de València

Congreso IN-RED (2018) 